\title{
Neutrino signature of Inert Doublet Dark Matter
}

\author{
Sarah Andreas \\ Institut für Theoretische Physik E, RWTH Aachen University, D-52056 Aachen, Germany \\ Service de Physique Théorique, Université Libre de Bruxelles, B-1050 Brussels, Belgium \\ Deutsches Elektronen-Synchrotron DESY, Notkestraße 85, D-22607 Hamburg, Germany ${ }^{1}$
}

\begin{abstract}
In the framework of the Inert Doublet Model and extensions, the signature of neutrinos from dark matter annihilation in the Earth, the Sun and at the Galactic centre is presented. The model contains an extra Higgs doublet, a neutral component of which is chosen as dark matter candidate. There are three distinct mass ranges for which consistency both with WMAP abundance and direct searches can be obtained: a low $(4-8 \mathrm{GeV})$, a middle $(60-70 \mathrm{GeV})$ and a high $(500-1500 \mathrm{GeV})$ WIMP mass range. The first case is of interest as we showed that the model can at the same time give the correct WMAP abundance and account for the positive DAMA results without contradicting other direct searches. We present how capture in the Sun can further constrain this scenario using Super-Kamiokande data. Indirect detection through neutrinos is challenging for the middle and high mass ranges. For the former, the presence of the so-called 'iron resonance' gives rise to larger neutrino fluxes for WIMP masses around 60 - $70 \mathrm{GeV}$ since capture by the Earth is enhanced. The addition of light right-handed Majorana neutrinos to the particle content of the model further increases the signal since it opens a direct annihilation channel into mono-energetic neutrinos. Neutrinos from the Galactic centre might be detected for heavy WIMPs if the dark matter density at the Galactic centre is substantially boosted.
\end{abstract}

Keywords: dark matter, astronomical observations of theories beyond the SM, neutrino experiments PACS: $95.35 .+\mathrm{d}$, 12.60.Fr, 95.85.Ry

\section{INTRODUCTION}

Driven by observations indicating that most matter in the universe is non-luminous, various dark matter candidates have been proposed. The candidate of the Inert Doublet Model (IDM) studied in this work belongs to the often studied category of weakly interacting massive particles (WIMPs) [1, 2]. Introduced in [3] as a simple standard model extension with an additional Higgs doublet and a scalar dark matter candidate, the IDM provides despite its simplicity a quite rich phenomenology. This has already been emphasized in several dedicated articles [4, 5, 6], investigations regarding LHC [7] and studies of the specific dark matter signatures which have been carried out for direct detection [8, 9] as well as indirect detection in gamma rays [10, 11], neutrinos [12, 13] and antimatter [14]. An interpretation of the IDM in a grand unified theory framework is given in [15].

In figure 1, we emphasize two examples of interesting features connected with recent or upcoming observations. It has been shown in [16, 17] that light WIMPs with spin-independent scattering on nuclei can account for the annual modulation signal reported by the DAMA collaboration [18] without contradicting the null results of the other direct detection experiments. In the framework of the IDM, it was found in [8] that agreement with DAMA can be obtained within the red regions of figure 1 (left plot) drawn in function of the dark matter mass $m_{H_{0}}$ and the only other relevant parameter of the model $\mu_{2}$ (the three regions result from the uncertainty on the Higgs to nucleus coupling $f$ ). Additionally, the abundance lies within the WMAP range between the two black lines, resulting in an overlap where both DAMA and WMAP can be fulfilled at the same time. Another test of this interesting scenario is possible with neutrinos from the Sun as will be discuss in this work. The right plot in figure 1 shows a "smoking gun" signal of monochromatic gamma-rays from the Galactic centre which has been presented in [11]. Those gamma lines result from a direct annihilation of the IDM dark matter candidate into $\gamma \gamma$ and $\gamma Z$, possible at loop-level and give a clear signal that might be observed with the upcoming Fermi satellite. The right relic density is obtained for those models due to a significant amount of coannihilations with the other inert particle $A_{0}$ of the model in the early Universe.

\footnotetext{
${ }^{1}$ Currently at DESY, email: Sarah.Andreas@desy.de
} 

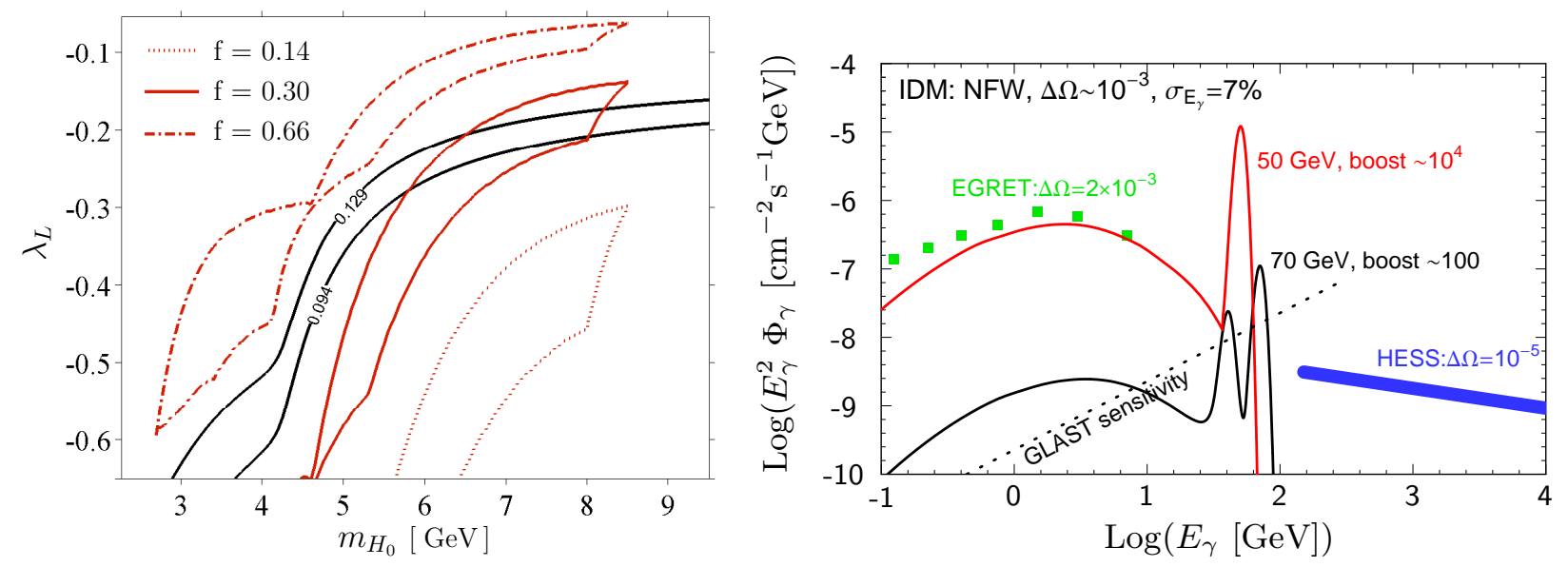

FIGURE 1. Features of the Inert Doublet model in view of recent or upcoming experiments:

left: Regions between the red lines can account for the DAMA annual modulation signal and nicely overlap with the one in which the correct WMAP abundance is obtained (between the black lines) [8].

right: Gamma lines from the annihilation of inert doublet dark matter into $\gamma \gamma$ and $\gamma Z$ (at loop-level) at the Galactic centre might be observed with the Fermi satellite whose sensitivity is shown as dotted line [11].

\section{THE INERT DOUBLET MODEL (IDM)}

The IDM is a standard model extension with two Higgs doublets $\left(H_{1}, H_{2}\right)$ and an unbroken $Z_{2}$ symmetry under which

$$
H_{1} \rightarrow H_{1} \text { and } H_{2} \rightarrow-H_{2}
$$

while all the other Standard Model particles are even. Its potential can be written as

$$
V=\mu_{1}^{2}\left|H_{1}\right|^{2}+\mu_{2}^{2}\left|H_{2}\right|^{2}+\lambda_{1}\left|H_{1}\right|^{4}+\lambda_{2}\left|H_{2}\right|^{4}+\lambda_{3}\left|H_{1}\right|^{2}\left|H_{2}\right|^{2}+\lambda_{4}\left|H_{1}^{\dagger} H_{2}\right|^{2}+\frac{\lambda_{5}}{2}\left[\left(H_{1}^{\dagger} H_{2}\right)^{2}+\text { h.c. }\right],
$$

where $H_{1}$ contains the Standard Model Higgs particle $h$. The discrete symmetry prevents flavour changing neutral currents and guarantees the stability of the lightest odd state. The lighter of the neutral components of the extra doublet $H_{2}=\left(H^{+}, 1 / \sqrt{2}\left(H_{0}+i A_{0}\right)\right)^{T}$ is therefore a candidate for dark matter and was in this work chosen to be $H_{0}$. We define $\lambda_{L}=\left(\lambda_{3}+\lambda_{4}+\lambda_{5}\right) / 2$ as the coupling between $h$ and a pair of $H_{0}$ and use as input parameters $\mu_{2}, \lambda_{2}$ together with the masses of the scalar particles, including the Higgs. We chose the parameters to be in agreement with the constraints discussed in [10] and experimental bounds from [5, 19, 20] and shade in the following figures those regions which are excluded by either of the two.

Under the assumption that $H_{0}$ was in thermal equilibrium in the early universe agreement of the abundance of $H_{0}$ (which we compute using micrOMEGAs [21]) with WMAP is found for a low ( $3 \mathrm{GeV} \lesssim m_{H_{0}} \lesssim 8 \mathrm{GeV}$ ), middle $\left(40 \mathrm{GeV} \lesssim m_{H_{0}} \lesssim 80 \mathrm{GeV}\right)$ and high mass $\left(500 \mathrm{GeV} \lesssim m_{H_{0}} \lesssim 1500 \mathrm{GeV}\right)$ range in agreement with [10, 22]. For each of those mass ranges a different annihilation channel is dominant. A light $H_{0}$ annihilates through the Higgs channel into fermions. In addition to this, if kinematically allowed, coannihilation with $A_{0}$ through a $Z$ boson takes place for the middle mass range. Annihilation into $W^{ \pm}$pairs opens above $80 \mathrm{GeV}$ and brings the abundance below WMAP because of its large cross section. Even though the annihilation cross section decreases and the relic abundance increases for higher masses, there is a parameter region consistent with WMAP around $1 \mathrm{TeV}[10]$.

A process relevant for the later discussion is the elastic scattering of $H_{0}$ on a nucleus $\mathscr{N}$ which is spin-independent in the framework of the IDM and occurs through Higgs exchange. Its low energy cross section is given by

$$
\sigma_{H_{0} \mathscr{N}_{i} \rightarrow H_{0} \mathscr{N}_{i}}^{S I}=\frac{1}{\pi} \frac{\lambda_{L}^{2}}{m_{h}^{4}} \frac{m_{\mathscr{N}_{i}}^{4}}{\left(m_{H_{0}}+m_{\mathscr{N}_{i}}\right)^{2}} f^{2},
$$

where $m_{\mathscr{N}_{i}}$ is the mass of the nucleus and $f$ is the nuclear form factor parameterizing the Higgs to nucleon coupling. This factor is related to the trace anomaly $f m_{\mathscr{N}} \equiv\left\langle\mathscr{N}\left|\sum_{q} m_{q} \bar{q} q\right| \mathscr{N}\right\rangle=g_{h \mathscr{N} \mathscr{N}} v$ but is unfortunately not well known. From the results quoted in [8], we take for a general nucleon $f=0.30$ as central value from the rather wide range $0.14<f<0.66$. 


\section{INDIRECT DETECTION}

Dark matter can be detected indirectly through the observation of its annihilation products among which the focus of this article lies on neutrinos. In general, the highest fluxes of annihilation products are expected to come from places with high dark matter density and accordingly high annihilation rate. Dark matter can accumulate over time in gravitational wells like the Galactic centre or get captured in astronomical bodies like the Sun or the Earth. Experiments like IceCube, Antares and Super-Kamiokande then search for secondary neutrinos from dark matter annihilation. Depending on the neutrino energy spectrum one distinguishes two extreme cases referred to as soft ( $v$ from $b \bar{b})$ and hard ( $v$ from $W$ and $Z$ ) spectra (the spectra of the different annihilation channels are computed with WimpSim [23, 24]). Detectors are more sensitive to the latter since a hard spectrum is flatter and has significant contributions at high energies. In the following, we present an overview of the relevant processes and formulas we used to obtain the results that will be presented in the next section.

\section{Dark matter annihilation at the Galactic centre}

A large amount of dark matter is expected to be concentrated around the Galactic centre thus opening the possibility for indirect detection through its annihilation products. Those fluxes of secondary particles strongly depend on the assumed dark matter density profile. Even though indications for a high dark matter density are also found in numerical simulations, the exact shape of this density profile especially close to the Galactic centre is still poorly known. At the Galactic centre, a flat behaviour is expected for a cored profile from observations of rotation curves [25, 26], while a more cuspy shape seems to be preferred by numerical simulations (see Kravtov et al. [26], Navarro-Frenk-White (NFW) [27] and Moore et al. [28] as well as the recent Via Lactea and AQUARIUS simulations [29, 30]). Due to this uncertainty boost factors are often considered in the analysis, expressing the fact that for higher dark matter densities larger fluxes can be expected. For instance, in [11], the predicted gamma flux is boosted by a factor $\sim 10^{4}$.

In addition to this astrophysical boost, the annihilation cross section for heavy dark matter candidates that interact through relatively lighter particles (for instance, the $W$, the $Z$ and the Higgs in the present case) might for low relative velocities be larger than the one at tree-level. This effect, called "Sommerfeld enhancement" [31, 32], might be nonnegligible and was found in models similar to the IDM like Minimal Dark Matter to be of $\mathscr{O}\left(10^{2}\right)$ at most [32, 33].

Despite those possibilities of boosting the flux, agreement with existing observational constraints for example in gamma rays must be maintained. Therefore, we follow a procedure proposed in [34] to circumvent the astrophysical uncertainties and to obtain an upper limit on the neutrino flux that might be reached either by a steeper dark matter profile and/or a Sommerfeld enhancement. The gamma ray spectrum from the Galactic centre is constrained by the observations of the EGRET satellite [35]. Requiring the predicted gamma flux to stay below the observed one at all energies (an energy of $E_{\gamma}=10 \mathrm{GeV}$ is found to be the most constraining), a maximal boost factor for a NFW profile is obtained for each point of the parameter space. The resulting boosts of $\sim 10^{2}-10^{3}$ are quite moderate considering that Sommerfeld enhancement might apply in addition to the astrophysical uncertainties. The maximum allowed neutrino flux is then computed according to

$$
\phi_{v}^{\max }=\phi_{v}^{N F W} \frac{\phi_{\gamma}^{E G R E T}(E=10 \mathrm{GeV})}{\phi_{\gamma}^{N F W}(E=10 \mathrm{GeV})} .
$$

\section{Capture and annihilation in the Sun/Earth}

Over time, astronomical bodies like the Sun or the Earth might also obtain a large dark matter density at their centre by accumulating WIMPs that are captured after having lost a part of their energy in elastic scattering on a nucleus. As discussed in the previous section, the coupling to nuclei is spin-independent in the IDM and the cross section depends on the mass of the nuclei, cf. equation (3). Since composition and size of Sun and Earth are different so is their ability to capture dark matter. The Sun is big and mainly composed out of Hydrogen which due to its small mass makes spin-dependent interactions with $\sigma \propto J(J+1)$ preferable. However, in the case of spin-independent interactions light particles are also well captured in the Sun. The much smaller Earth consists dominantly of heavy nuclei and therefore capture is strong for spin-independent interactions.

Our calculation of the capture rate is based on an elementary treatment and follows the simple approximation of [1,36] which is expected to work for the Sun but not necessarily for the Earth since the escape velocity of the Earth 
is small relative to the mean velocity in the halo making capture in the Earth sensitive to the (not well understood) small velocity part of the distribution. In [1], the Earth is treated as if it was in free space, while in general the gravitational field of the Sun would have to be taken into account. However, it has been found that the approximations of [1] work reasonably well for dark matter masses close to the iron resonance ${ }^{2}$, which is just the interest of this work.

The capture rate in the Sun/Earth for spin-independent scattering on nuclei is then given by

$$
C^{\odot / \oplus}=c_{\odot / \oplus} \frac{\rho_{\text {local }}}{0.3 \mathrm{GeV} \mathrm{cm}^{-3}} \frac{270 \mathrm{~km} / \mathrm{s}}{\bar{v}_{\text {local }}} \sum_{i} F_{i} f_{i} \phi_{i} S_{i} \frac{\mathrm{GeV}^{2}}{m_{\mathscr{N}_{i}} m_{H_{0}}} \frac{\sigma_{i}}{10^{-40} \mathrm{~cm}^{2}},
$$

where $c_{\odot}=4.8 * 10^{24} \mathrm{~s}^{-1}\left(c_{\oplus}=4.8 * 10^{15} \mathrm{~s}^{-1}\right)$ sets the scale for WIMP capture by the Sun (Earth), cf. ref. [1] and $\sigma_{i}$ has to be taken from equation (3). The local dark matter density and mean velocity are set to the typical values $\rho_{\text {local }}=0.3 \mathrm{GeV}$ and $\bar{v}_{\text {local }}=270 \mathrm{~km} / \mathrm{s}$. In the summation over all elements in the Sun/Earth, we use the values given in [1] for the mass fraction $f_{i}$ and the distribution $\phi_{i}$ of element $i$. The kinematics of the collision are encoded in the nuclear form factors $F_{i}=F_{i}\left(m_{H_{0}}\right)$ and the suppression factor $S_{i}=S_{i}\left(\frac{m_{H_{0}}}{m_{N_{i}}}\right)$, both defined in [1], where also a detailed discussion of the behaviour of the capture rate as a function of the dark matter mass can be found.

In the following, we will consider dark matter with a mass close to the one of iron thereby taking advantage of the so called iron resonance which enhances the capture rate in the Earth. For details about the origin of this and other similar resonances please refer to [1, 12]. From simple kinematics, it is known that the energy loss of a WIMP is largest and therefore capture most efficient if it scatters on a nucleus with similar mass. Since iron is abundant in the Earth, the capture rate is large for WIMPs with masses around $60 \mathrm{GeV}$.

Once captured, dark matter annihilates at the centre of the Sun/Earth producing a flux of secondary particles $i$ given by

$$
\left(\frac{d \phi}{d E}\right)_{i}=\frac{1}{2} \frac{C F_{\mathrm{EQ}}}{4 \pi R^{2}} \sum_{F} B R_{F}\left(\frac{d N}{d E}\right)_{F, i},
$$

where $C$ is the capture rate, cf. equation $(5), R$ is either the distance from the Sun to the Earth $\left(R=d_{\odot-\oplus}=\right.$ $\left.1.5 * 10^{8} \mathrm{~km}\right)$ or the radius of the Earth $\left(R=r_{\oplus}=6300 \mathrm{~km}\right)$ and $F_{E Q}$ is a factor defined in [1] describing whether or not equilibrium between capture and annihilation has been established. The sum is carried out over all kinematically allowed final states $F$ with branching ratio $B R_{F}$ and differential spectrum $(d N / d E)_{F, i}$ of annihilation products $i$.

Neutrinos from dark matter annihilation at the Sun or the Earth can be observed with neutrino telescopes such as IceCube or Super-Kamiokande. In general the best directional information is obtained for muonic neutrinos by detection of the muon which is produced in a charged-current interactions in the rock below the detector. Therefore, in the following, we focus on muon-neutrinos and present the detection rate of neutrino-induced, through-going muon events from the Sun or the Earth given by

$$
\phi_{\mu}^{\odot / \oplus}=\varphi_{\odot / \oplus} \frac{C^{\odot / \oplus} F_{\mathrm{EQ}}}{2 \mathrm{~s}^{-1}}\left(\frac{m_{H_{0}}}{\mathrm{GeV}}\right)^{2} \sum_{i} a_{i} b_{i} \sum_{F} B R_{F}\left\langle N z^{2}\right\rangle_{F, i}\left(m_{H_{0}}\right),
$$

where $\varphi_{\odot}=2.54 * 10^{-23} \mathrm{~km}^{-2} \mathrm{yr}^{-1}$ and $\varphi_{\oplus}=\varphi_{\odot}\left(d_{\odot-\oplus} / r_{\oplus}\right)^{2}=\varphi_{\odot} * 5.6 * 10^{6}$, cf. [1]. The neutrino-scattering coefficients $a_{i}$ and muon-range coefficients $b_{i}$ are given in [1] as $a_{v}=6.8, a_{\bar{v}}=3.1, b_{v}=0.51$ and $b_{\bar{v}}=0.67$. The second moment of the muon-neutrino spectrum $\left\langle N z^{2}\right\rangle_{F, i}\left(m_{H_{0}}\right)$ scaled by the square of the injection energy $E_{\text {in }}$ is

$$
\left\langle N z^{2}\right\rangle_{F, i}\left(E_{\text {in }}\right)=\frac{1}{E_{\text {in }}^{2}} \int\left(\frac{d N}{d E}\right)_{F, i}\left(E_{v}, E_{\text {in }}\right) E_{v}^{2} d E_{v}
$$

where the integration contains as lower bound the threshold energy of the detector.

\section{IDM NEUTRINO SIGNATURES}

In this section, our results on the neutrino fluxes from the annihilation of inert doublet dark matter are presented. From the discussion of the previous sections follows that in each of the three mass ranges where the abundance is

${ }^{2}$ Detailed investigations of this subject can be found in [37, $\left.38,39,40,41,42\right]$. 
in agreement with WMAP it is most interesting to study neutrinos from one specific origin. For heavy dark matter candidates, capture in the Sun or the Earth is suppressed because of the $1 / m_{D M}^{2}$ dependence of the capture cross section (3) designating the Galactic centre as the most promising place to observe. Dark matter candidates of the middle range have a mass close to the iron resonance and therefore experience a strong enhancement of their capture rate in the Earth. The lightest IDM candidates do not benefit from resonances but are best captured in the Sun due to the $1 / m_{D M}^{2}$ dependence favouring light particles.

\section{Galactic centre and heavy dark matter}

The most promising signal from the annihilation of dark matter in the mass range $500 \mathrm{GeV} \lesssim m_{H_{0}} \lesssim 1500 \mathrm{GeV}$ is expected to come from the Galactic centre. For such heavy $H_{0}$ candidates, it has been argued in ref. [10] that only $m_{H_{0}} \sim \mu_{2}$ gives the correct WMAP abundance and is in agreement with model constraints (vacuum stability and perturbativity). In this region of the parameter space, annihilation occurs dominantly into $W$ and $Z$ bosons, both producing hard neutrinos.

In figure 2, we show the upper limit on the expected neutrino flux in Antares from the annihilation of $H_{0}$ at the Galactic centre computed according to equation (4). All points in the scatter plot are consistent with model constraints. The dark blue ones agree within $2 \sigma$ with WMAP while the light blue ones give too high or too low abundance. The sensitivity of Antares taken from [43] is given both for a hard (solid red line) and a soft (dashed red line) neutrino spectrum. Since the neutrinos result mainly from $W$ and $Z$ decay their spectrum can be considered hard and should be compared to the solid sensitivity line. The maximum flux then lies above the Antares sensitivity for $H_{0}$ masses above $900 \mathrm{GeV}$ and might potentially lead to a signal. For several models in this range, the dark matter abundance is in agreement with WMAP.

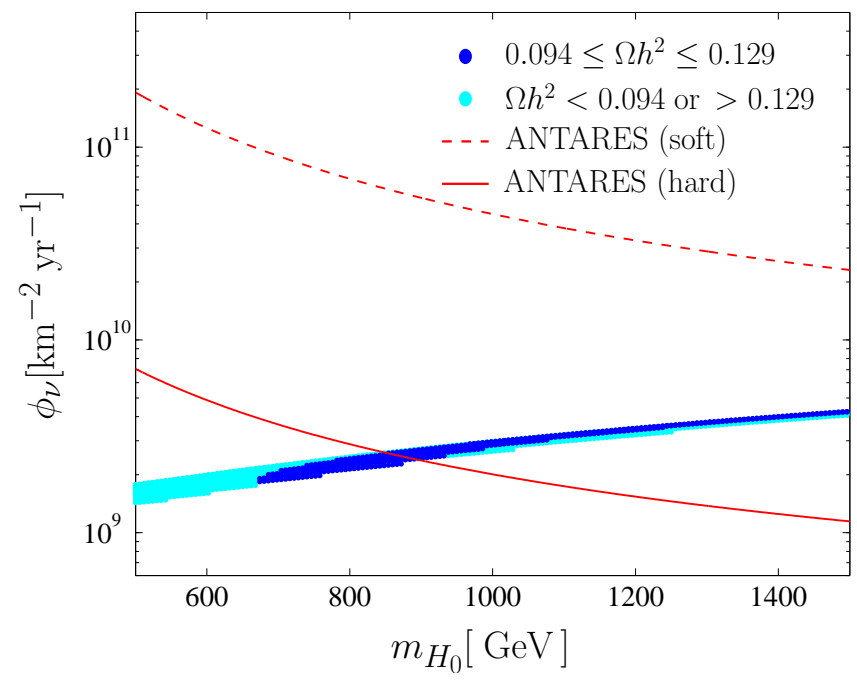

FIGURE 2. Scatter plot of the maximum expectable neutrino flux in the Antares detector for the IDM for which the corresponding $\gamma$-flux does not exceed the one observed by EGRET. The sensitivities of Antares for a soft (dashed) and a hard (solid) spectrum are shown in red. The dark blue points lie within the WMAP area while the light blue ones are outside. (Parameters: $m_{h}=120 \mathrm{GeV}$, $\left.\lambda_{2}=0.2, \Delta m_{A_{0} H_{0}}=5 \mathrm{GeV}, \Delta m_{H^{+} H_{0}}=10 \mathrm{GeV}\right)$.

\section{Earth and iron mass range}

As discussed earlier, the Earth captures efficiently dark matter with a mass close to the one of iron thus providing an interesting place to search for a signal. An upper estimate on the expected muon events in IceCube obtained from equations (7) and (8) without applying detector thresholds is plotted in figure 3 in function of the dark matter mass $m_{H_{0}}$ and the bare mass scale $\mu_{2}$ for two different Higgs masses. The shaded parts are excluded, the black lines give the $2 \sigma$ WMAP region and the blue ones the sensitivity of IceCube. In the considered dark matter mass range, neutrinos 
come mainly from $b \bar{b}$ and have to be compared to the sensitivity for a soft neutrino spectrum. Direct detection (white lines, XENON and CDMS [44]) strongly constrains the parameter space and excludes all regions for which the correct abundance and a flux above the sensitivity is obtained. Our results are in agreement with those of [13].

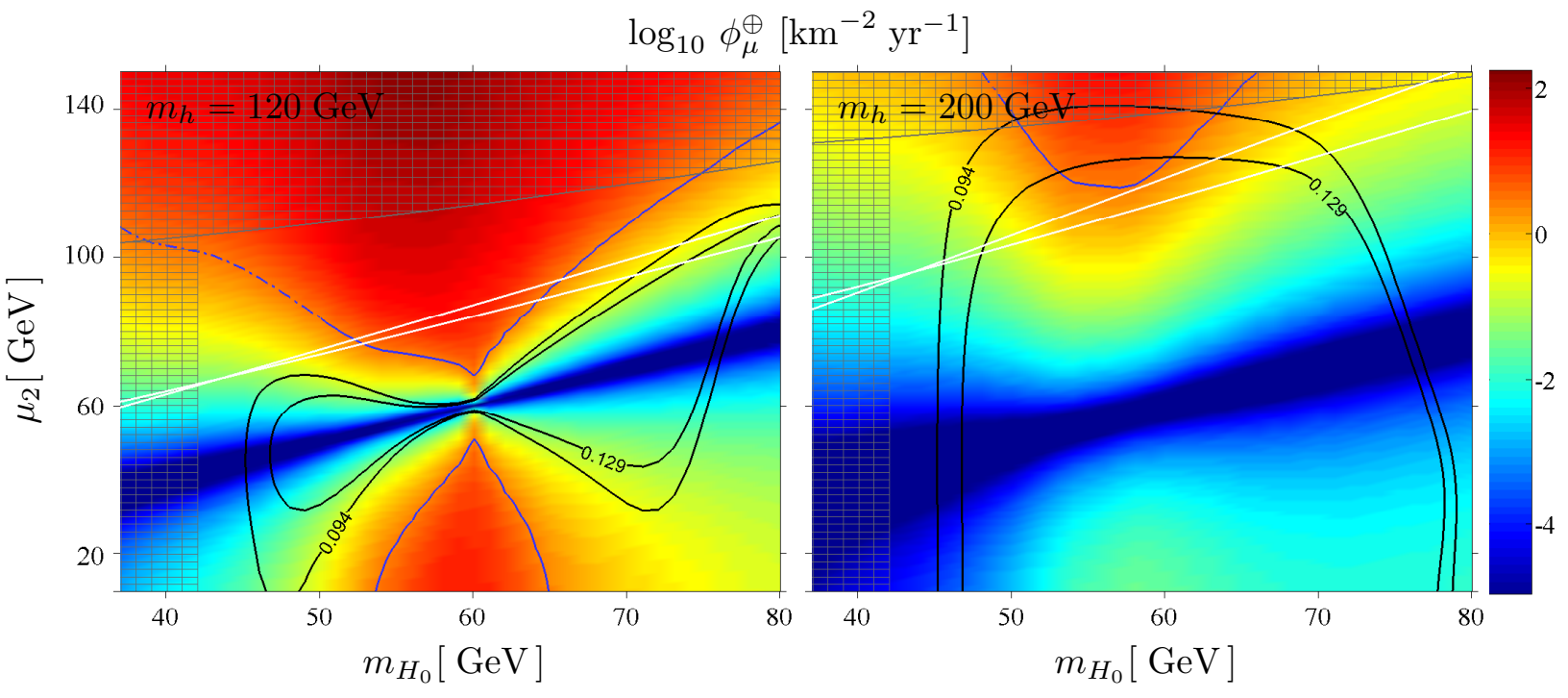

FIGURE 3. Upper estimate of the muon flux in IceCube (without detector threshold) from annihilation at the Earth. Colour gradient - $\log _{10} \phi_{\mu}\left[\mathrm{km}^{-2} \mathrm{yr}^{-1}\right]$; WMAP area - black lines; XENON, CDMS limits - white lines; IceCube sensitivity soft - blue lines; excluded regions - shaded. (Parameters: $\lambda_{2}=0.2, \Delta m_{A_{0} H_{0}}=8 \mathrm{GeV}, \Delta m_{H^{+} H_{0}}=50 \mathrm{GeV}, f=0.3$ )

In the following, we discuss two possibilities that might improve the prospects for IceCube. Recent numerical simulations indicated the presence of a disc consisting of dark matter in our Galaxy [45, 46]. Since the relative velocity of WIMPs in the dark disc compared to the solar system is smaller then for usual WIMPs from the halo, the capture rate in the Earth would be enhanced. The authors find in their study which is performed in the framework of the MSSM that the muon fluxes from the Earth might then be boosted by a factor 10-100 [47]. A similar enhancement applied to the IDM gives a more promising prediction for the muon fluxes in IceCube.

An extension of the model with three right-handed Majorana neutrinos that has been proposed in [4] in order to generate neutrino masses through radiative corrections is also interesting in terms of neutrino fluxes. It opens a new annihilation channel for $H_{0}$ directly into neutrinos as shown in figure 5 thereby producing a monochromatic flux. The IDM Lagrangian must be enlarged by the new terms

$$
\mathscr{L} \supset h_{i j}\left(v_{i} H_{0}-l_{i} H^{+}\right) N_{j}+\frac{1}{2} M_{j} N_{j} N_{j}+\text { h.c. }
$$

where the index $i$ stands for the SM neutrino generation $(i=e, \mu, \tau)$ and $j$ for the three extra Majorana neutrinos $(j=1,2,3)$. Even though the Majorana neutrinos are also odd under $Z_{2}$ we keep $H_{0}$ as dark matter candidate and find

$$
\sigma|\vec{v}|=\frac{h^{4}}{4 \pi} \frac{m_{N}^{2}}{\left(m_{H_{0}}^{2}+m_{N}^{2}\right)^{2}}
$$

for its annihilation cross section into $v v$ or the conjugate channel $\overline{v v}$. For simplicity, we focus on muon-neutrinos and suppose that only one Majorana neutrino, with mass $m_{N}$, enters the cross section. Also we do not take into account neutrino oscillations, which is a good approximation for the Earth. We take the Majorana neutrino to be rather light $\left(m_{N}=100 \mathrm{GeV}\right)$ and $h=0.1$ in order to get a large neutrino signal.

In figure 4. we give the muon flux from mono-energetic neutrinos for different Higgs masses together with the IceCube sensitivity for a hard neutrino spectrum (blue lines). Direct detection constraints (white lines) are the same as in the previous section, however the WMAP region slightly changes shape due to the additional annihilation channel into neutrinos. Unless the Higgs is light $(120 \mathrm{GeV})$ there exists a region of the parameter space for which the muon fluxes are above the IceCube sensitivity and agreement with WMAP and direct detection is obtained.

This result is also illustrated in the scatter plot in figure 5 which gives the muon flux from the Earth in function of the dark matter mass together with the sensitivities of several experiments. The expected muon fluxes for the MSSM are 
additionally shown for comparison. The fluxes for the IDM are typically larger than for the MSSM and lie around the iron resonance well above the IceCube sensitivity while also agreeing with WMAP and direct detection constraints. A light Majorana particle as needed for high fluxes on the negative side gives large one-loop contributions to the SM neutrino masses $(\sim \mathrm{keV})$ whose cancelation requires some fine tuning as discussed in [12].

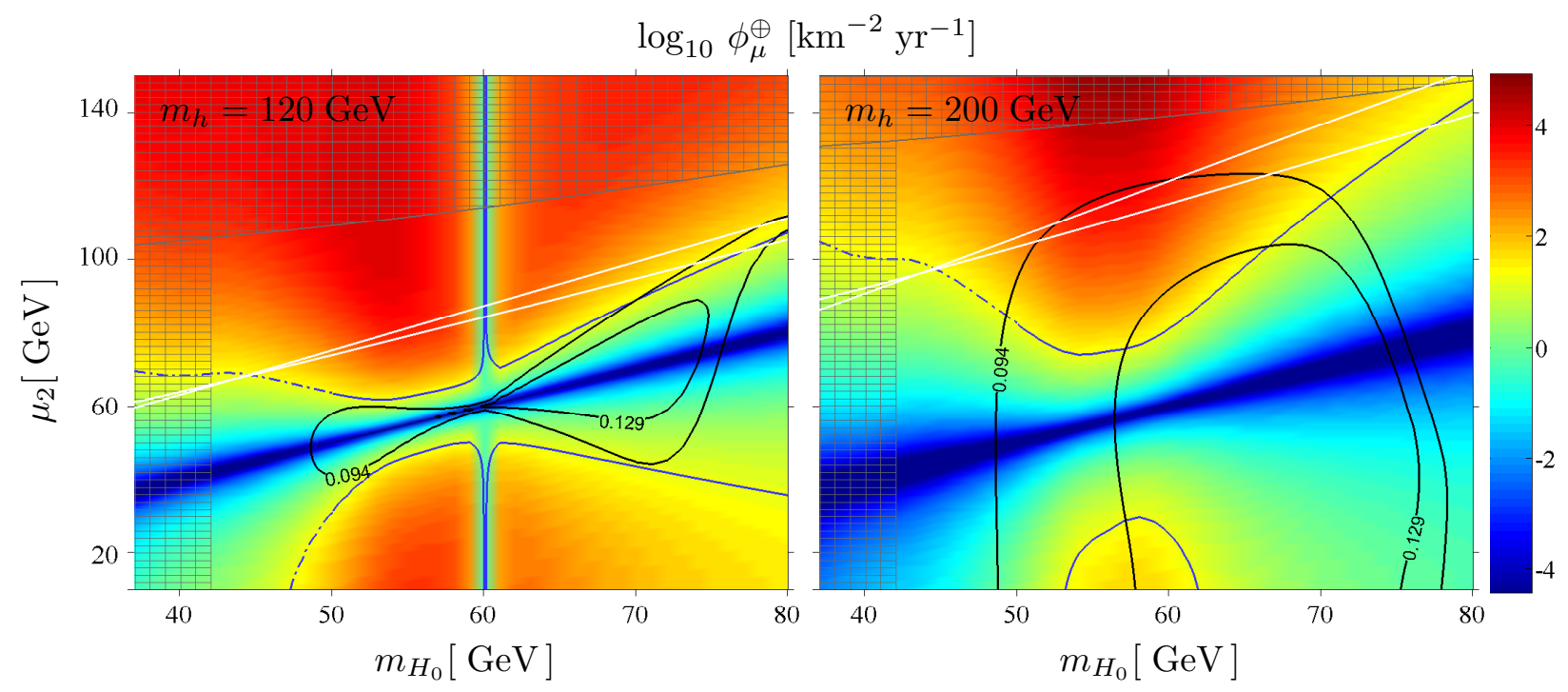

FIGURE 4. Muon flux from the Earth for the IDM extended with light Majorana neutrinos opening annihilation into monoenergetic neutrinos. Colour gradient - $\log _{10} \phi_{\mu}\left[\mathrm{km}^{-2} \mathrm{yr}^{-1}\right]$; WMAP area - black lines; XENON, CDMS limits - white lines; IceCube sensitivity hard - blue lines; excluded regions - shaded. (Parameters: $\lambda_{2}=0.2, \Delta m_{A_{0} H_{0}}=8 \mathrm{GeV}, \Delta m_{H^{+} H_{0}}=50 \mathrm{GeV}$, $\left.m_{N}=100 \mathrm{GeV}, h=0.1, f=0.3\right)$.
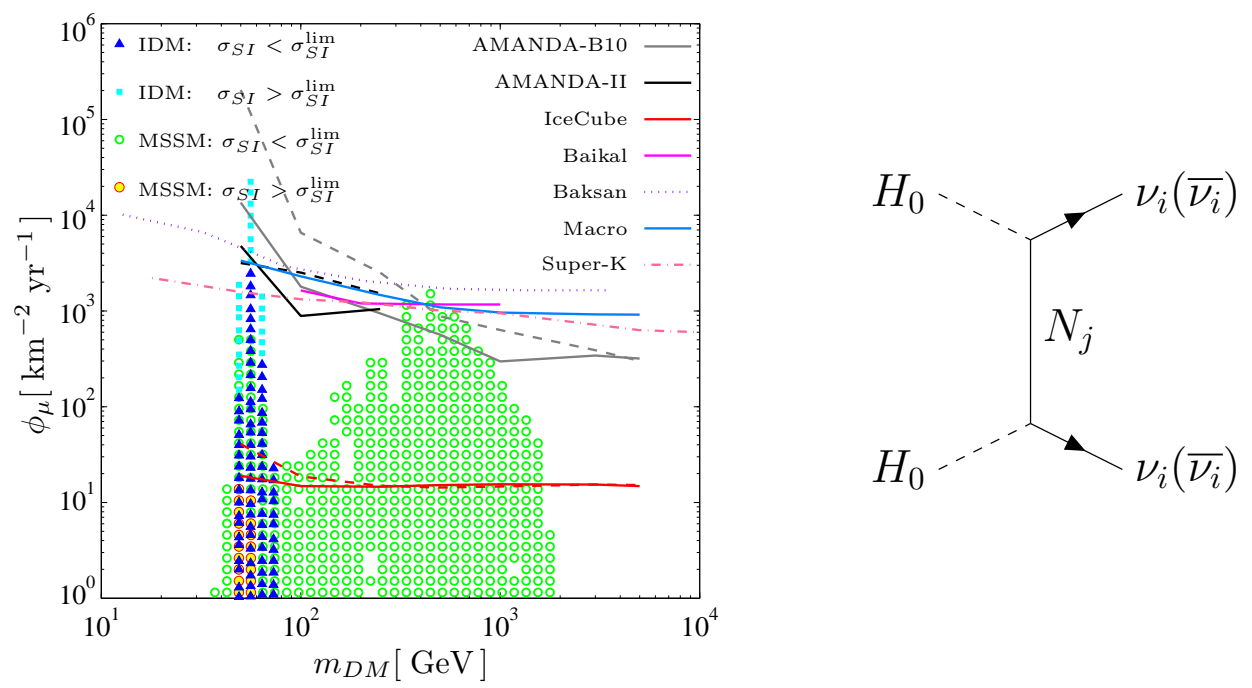

FIGURE 5. left: Scatter plot of the muon fluxes from the Earth for the IDM with Majorana extension (dark blue below, light blue above direct detection limit) and the MSSM (yellow below, green above direct detection limit). All plotted points are within the $3 \sigma$ WMAP region. Lines correspond to detector thresholds: AMANDA-B10 (1997-1999) hard (solid) and soft (dashed); AMANDA-II (2001-2003) hard (solid) and soft (dashed); IceCube best case hard (solid) and soft (dashed); BAIKAL (1998-2001); BAKSAN (1978-1995); MACRO (1989-1998) hard; SUPER-K (1996-2001) soft, see ref. [48] and references therein. (Parameters: $\left.m_{h}=200 \mathrm{GeV}, \lambda_{2}=0.2, \Delta m_{A_{0} H_{0}}=8 \mathrm{GeV}, \Delta m_{H^{+} H_{0}}=50 \mathrm{GeV}, f=0.3, m_{N}=100 \mathrm{GeV}, h=0.1\right)$.

right: Direct annihilation of $H_{0}$ through exchange of a Majorana neutrino $N_{j}$ into neutrinos $v_{i}$ (or antineutrinos $\bar{v}_{i}$ ). 


\section{Sun and light dark matter}

As presented above, the light IDM dark matter candidate has been studied in [8] in view of explaining the DAMA annual modulation signature. In this reference, it has been shown that a large gamma flux from the Galactic centre might be studied with the Fermi satellite. The most promising neutrino signal however is expected to come from the Sun which captures light WIMPs well.

In figure 6, we show both the flux of muon-neutrinos computed with equation (6) and that of upgoing muons from equation (7) as a function of the dark matter mass $m_{H_{0}}$ and the bare mass scale $\mu_{2}$. The WMAP allowed region is given in black and current direct detection constraints in white (from left to right XENON and CDMS [44]). In the right figure of the muon flux, the DAMA allowed region according to figure 1 for $f=0.3$ is drawn in addition (dashed magenta lines).

Since the neutrinos resulting from such a light dark matter candidate have a quite low energy, they might only be constrained by the Super-Kamiokande experiment if its sensitivity which currently reaches down to $m_{D M}=18 \mathrm{GeV}$ is extended to even lower masses. This might be achieved including stopping, partially and fully contained muons [49]. In figure 6, we draw in blue an estimate of this sensitivity obtained by horizontally extrapolating the one given in [50] to lower masses (rescaled to a muon threshold of $1 \mathrm{GeV}$, cf. [48]).

It can be seen that the light IDM candidate and a part of the DAMA allowed region might be tested by SuperKamiokande but only for a parameter region in which the coupling $\lambda_{2}$ is required to be rather $\operatorname{larg} \mathrm{f}^{3}\left(\lambda_{2}>1\right.$ in the shaded regions). A similar conclusion is presented in [8], cf. figure 5 therein, by adapting the analysis of [51].

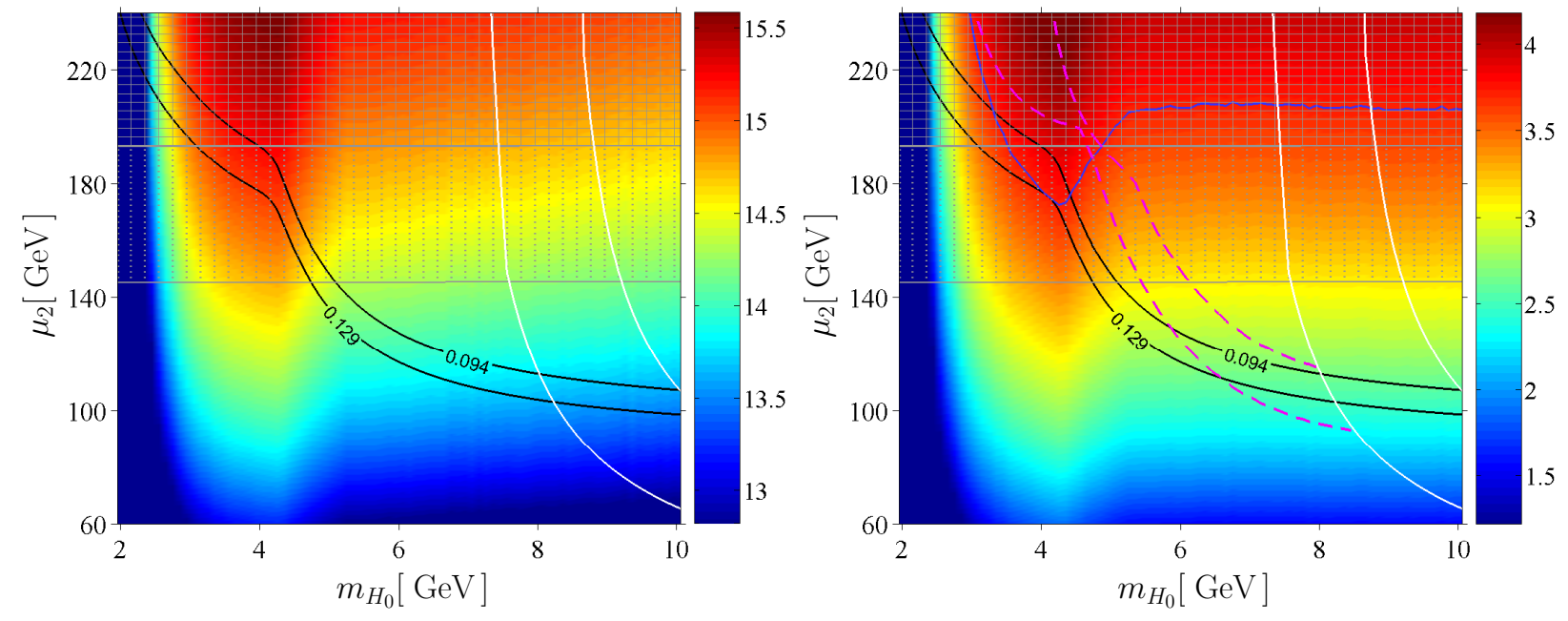

FIGURE 6. Neutrino (left) and muon (right) fluxes from the Sun together with our conservative low energy extrapolation of the Super-Kamiokande sensitivity and the DAMA region in the right plot. Colour gradient $-\log _{10} \phi_{v}\left[\mathrm{~km}^{-2} \mathrm{yr}^{-1}\right](l e f t)$ and $\log _{10} \phi_{\mu}\left[\mathrm{km}^{-2} \mathrm{yr}^{-1}\right]$ (right); WMAP area - black lines; DAMA allowed region - dashed magenta lines; XENON, CDMS limits - white lines, from left to right; shaded regions correspond to $\lambda_{2}>1$. (Parameters: $m_{h}=120 \mathrm{GeV}, \lambda_{2}=1$ (dotted) and $\lambda_{2}=\pi$ (shaded), $f=0.3$ ).

\section{CONCLUSIONS}

We presented the signature of the Inert Doublet Model in indirect detection of neutrinos in three distinct mass ranges and each from a different place of origin: the Galactic centre, the Earth and the Sun. For dark matter masses around $1 \mathrm{TeV}$ we predict an upper limit on the neutrino flux so that the corresponding gamma flux stays compatible with the one observed by EGRET. With a boost of $\mathscr{O}\left(10^{2}-10^{3}\right)$ from the astro- (dark matter profile) and/or particle physics (Sommerfeld enhancement) side, this flux might be observed in Antares. Prospects for the Earth and WIMPs with

\footnotetext{
${ }^{3}$ As discussed in [8], in order to bring $m_{H_{0}}$ and $\mu_{2}$ in the range of WMAP and DAMA while ensuring the stability of the potential one needs $\lambda_{2}>2\left(m_{H_{0}}^{2}-\mu_{2}^{2}\right)^{2} /\left(v^{2} m_{h}^{2}\right)$.
} 
a mass $\sim 60 \mathrm{GeV}$ are strongly constrained by limits from direct detection experiments. Even though those WIMPs experience an enhancement of their capture rate in the Earth due to the iron resonance, we found no solutions with an observable flux in IceCube and agreement both with the WMAP abundance and direct detection limits. This result agrees with the study performed in [13]. Assuming the existence of a dark disc in our Galaxy as indicated by numerical simulations might however give a sizeable boost $\mathscr{O}(10-100)$ to the predicted fluxes leading to a more promising prediction. An extension of the model with light Majorana neutrinos has also been shown to give a potential signal in IceCube due to the production of mono-energetic neutrinos via a new annihilation channel. Finally, for the light dark matter candidate with a mass of a few $\mathrm{GeV}$, we consider the parameter region that was previously found to be consistent with the DAMA signal. In agreement with [51, 52], we show that neutrinos from the Sun can be used to further test this scenario with Super-Kamiokande.

\section{ACKNOWLEDGMENTS}

This work has been done in collaboration with Michel H. G. Tytgat.

\section{REFERENCES}

1. G. Jungman, M. Kamionkowski, and K. Griest, Phys. Rept. 267 (1996) 195-373, arXiv : hep-ph/9506380.

2. G. Bertone, D. Hooper, and J. Silk, Phys. Rept. 405 (2005) 279-390|arXiv : hep-ph/ 0404175

3. N. G. Deshpande and E. Ma, Phys. Rev. D18 (1978) 2574.

4. E. Ma, Phys. Rev. D73 (2006) 077301 arXiv: hep-ph/0601225

5. R. Barbieri, L. J. Hall, and V. S. Rychkov, Phys. Rev. D74 (2006) 015007, arXiv: hep-ph/ 0603188.

6. T. Hambye and M. H. G. Tytgat, Phys. Lett. B659 (2008) 651-655 arXiv:0707.0633 [hep-ph]

7. E. Dolle, X. Miao, S. Su, and B. Thomas, arXiv:0909.3094 [hep-ph]

8. S. Andreas, T. Hambye, and M. H. G. Tytgat, JCAP 0810 (2008) 034, arXiv: 0808.0255 [hep-ph].

9. C. Arina, F.-S. Ling, and M. H. G. Tytgat, arXiv:0907.0430 [hep-ph]

10. L. Lopez Honorez, E. Nezri, J. F. Oliver, and M. H. G. Tytgat, JCAP 0702 (2007) 028, arXiv: hep-ph/0612275

11. M. Gustafsson, E. Lundstrom, L. Bergstrom, and J. Edsjo, Phys. Rev. Lett. 99 (2007) 041301|arXiv : astro-ph/0703512

12. S. Andreas, M. H. G. Tytgat, and Q. Swillens, JCAP 0904 (2009) 004 arXiv: 0901.1750 [hep-ph]

13. P. Agrawal, E. M. Dolle, and C. A. Krenke, Phys. Rev. D79 (2009) 015015 arXiv: 0811.1798 [hep-ph]

14. E. Nezri, M. H. G. Tytgat, and G. Vertongen, JCAP 0904 (2009) 014 arXiv: 0901.2556 [hep-ph]

15. M. Kadastik, K. Kannike, and M. Raidal, Phys. Rev. D80 (2009) 085020||arXiv: 0907.1894 [hep-ph]

16. F. Petriello and K. M. Zurek, JHEP 09 (2008) 047, arXiv: 0806.3989 [hep-ph]

17. C. Savage, G. Gelmini, P. Gondolo, and K. Freese, JCAP 0904 (2009) 010, arXiv: 0808.3607 [astro-ph]

18. DAMA Collaboration, R. Bernabei et al., Eur. Phys. J. C56 (2008) 333-355, arXiv: 0804.2741 [astro-ph]

19. E. Lundstrom, M. Gustafsson, and J. Edsjo, Phys. Rev. D79 (2009) 035013, arXiv: 0810.3924 [hep-ph]

20. Q.-H. Cao, E. Ma, and G. Rajasekaran, Phys. Rev. D76 (2007) 095011, arXiv: 0708.2939 [hep-ph]

21. G. Belanger, F. Boudjema, A. Pukhov, and A. Semenov, Comput. Phys. Commun. 176 (2007) 367-382 arXiv:hep-ph/0607059.

22. E. M. Dolle and S. Su, Phys. Rev. D80 (2009) 055012, arXiv:0906.1609 [hep-ph]

23. J. Edsjö, WimpSim Neutrino Monte Carlo, http://www.physto.se/ edsjo/wimpsim/.

24. M. Blennow, J. Edsjo, and T. Ohlsson, JCAP 0801 (2008) 021. arXiv: 0709.3898 [hep-ph]

25. R. A. Flores and J. R. Primack, Astrophys. J. 427 (1994) L1-4, arXiv: astro-ph/9402004

26. A. V. Kravtsov, A. A. Klypin, J. S. Bullock, and J. R. Primack, Astrophys. J. 502 (1998) 48, arXiv:astro-ph/9708176

27. J. F. Navarro, C. S. Frenk, and S. D. M. White, Astrophys. J. 462 (1996) 563-575, arXiv:astro-ph/9508025

28. B. Moore et al., Astrophys. J. 524 (1999) L19-L22.

29. J. Diemand et al., Nature 454 (2008) 735-738 arXiv: 0805.1244 [astro-ph]

30. V. Springel et al., Mon. Not. Roy. Astron. Soc. 391 (2008) 1685-1711, arXiv:0809.0898 [astro-ph]

31. J. Hisano, S. Matsumoto, M. M. Nojiri, and O. Saito, Phys. Rev. D71 (2005) 063528, arXiv: hep-ph/0412403

32. M. Cirelli, A. Strumia, and M. Tamburini, Nucl. Phys. B787 (2007) 152-175 arXiv:0706.4071 [hep-ph]

33. M. Cirelli, R. Franceschini, and A. Strumia, Nucl. Phys. B800 (2008) 204-220 arXiv: 0802.3378 [hep-ph]

34. G. Bertone, E. Nezri, J. Orloff, and J. Silk, Physical Review D 70 (2004) 063503 arXiv: astro-ph/ 0403322.

35. S. D. Hunter et al., The Astrophysical Journal 481 (May, 1997) 205-240

36. W. H. Press and D. N. Spergel, Astrophys. J. 296 (1985) 679-684

37. A. Gould, Astrophys. J. 328 (1988) 919-939

38. A. Gould and S. M. Khairul Alam, Astrophys. J. 549 (2001) 72-75, arXiv: astro-ph/9911288

39. T. Damour and L. M. Krauss, Phys. Rev. Lett. 81 (1998) 5726-5729 arXiv: astro-ph/980 6165

40. J. Lundberg and J. Edsjo, Phys. Rev. D69 (2004) 123505 arXiv:astro-ph/ 0401113. 
41. A. H. G. Peter, Phys. Rev. D79 (2009) 103531, arXiv:0902.1344 [astro-ph.HE]

42. A. H. G. Peter, Phys. Rev. D79 (2009) 103533, arXiv: 0902.1348 [astro-ph.HE]

43. D. J. Bailey, Monte Carlo tools and analysis methods for understanding the ANTARES experiment and predicting its sensitivity to Dark Matter. PhD thesis, Wolfson College, Oxford, 2002.

44. CDMS-Collaboration, Phys. Rev. Lett. 102(2009) 011301, arXiv:0802.3530 [astro-ph]

45. J. I. Read, G. Lake, O. Agertz, and V. P. Debattista, arXiv:0803.2714 [astro-ph]

46. T. Bruch, J. Read, L. Baudis, and G. Lake, Astrophys. J. 696 (2009) 920-923, arXiv: 0804.2896 [astro-ph]

47. T. Bruch, A. H. G. Peter, J. Read, L. Baudis, and G. Lake, Phys. Lett. B674 (2009) 250-256, arXiv: 0902.4001 [astro-ph.HE]

48. IceCube Collaboration, D. Hubert and A. Davour, "Search for neutralino dark matter with the AMANDA neutrino telescope," in 30th International Cosmic Ray Conference (ICRC 2007). 2007.

49. J. L. Feng, J. Kumar, J. Learned, and L. E. Strigari, JCAP 0901 (2009) 032 arXiv:0808.4151 [hep-ph]

50. Super-Kamiokande Collaboration, S. Desai et al., Phys. Rev. D70 (2004) 083523|arXiv: hep-ex/0404025

51. D. Hooper, F. Petriello, K. M. Zurek, and M. Kamionkowski, Phys. Rev. D79 (2009) 015010. arXiv:0808.2464 $[$ hep-ph].

52. J. L. Feng, J. Kumar, and L. E. Strigari, Phys. Lett. B670 (2008) 37-40 arXiv:0806.3746 [hep-ph]. 